\title{
Physical activity recognition via minimal in-shoes force sensor configuration
}

\author{
Christopher Moufawad el Achkar*, Fabien Massé, Arash Arami, Kamiar Aminian \\ Laboratory of Movement Analysis and Measurement \\ École Polytechnique Fédérale de Lausanne \\ Lausanne, Switzerland
}

\begin{abstract}
We propose a new minimal wearable system and a classifier for physical activity recognition. The configuration is solely based on two force sensors placed anteriorly and posteriorly under the feet. To find the optimal sensor configuration, we estimated the total force under the feet during daily activities. The estimation was based on a linear regression model built upon the forces estimated over selected areas from the dense mesh of high-resolution sensors of a commercially-available force sensing system. The best estimate of the total force, which also indicated the best sensor configuration, was fed to the activity recognition algorithm to provide the final output. The analysis indicated that the optimal locations which allowed estimating the total force with a minimal RMS error (40N) were the central part of rear foot and forefoot. Using this configuration and the activity classification algorithm, the classification accuracy for the basic activities such as sitting, standing and walking were 93.8\%, 99.5\% and $93.4 \%$, respectively. These values demonstrate the high accuracy of the proposed system and are very encouraging for recognition of additional types of activities of daily-living in the next stage.
\end{abstract}

Keywords-plantar force; minimal sensor configuration; activity classification

*email : christopher.moufawadelachkar@epfl.ch

\section{INTRODUCTION}

Long-term monitoring of physical activity has witnessed significant advances in recent years. This has been accelerated by the increasing availability of cheap and light wearable sensors. A decline in physical activity levels is considered as a precursor for diseases and functional impairments [1]. Plantar force information is valuable for accurate activity recognition using wearable systems [2]. Plantar force and pressure distribution measurements have been conducted in relation to rehabilitation, fall prevention, assessment of diseases such as arthritis, sports and robotics [3].

Plantar force measurements have been performed in various ways, including force plates, floor mats, insoles and pressure/force load cells. Force plates or mats yield accurate results for the total force experienced during standing and walking [4]. However, only one or a few steps can be analyzed therefore these instruments are not suitable for long-term evaluation of forces under the feet. Wearable systems such as insoles and standalone sensors provide an alternative with acceptable validity for such measurements. Indeed, many insoles containing multiple force sensors rival floor-fixed or mat sensors in terms of accuracy. These insoles are expensive due to the high spatial resolution of individual sensors. Another issue is the size and location of the sensors that should be used. Many studies indicated that overlaying a certain number of cells under the foot is not necessarily ideal since many cells are not activated during physical activity (PA) episodes [5], [6]. Standalone force sensing resistors (FSR) have been used in previous shoebased designs for PA monitoring [2], [7], [8] but conclusive results on the number, size, shape and location of sensors to be used were not readily available. The conclusion is that there is a need for a cheap and easy to use foot worn solution for force sensing in daily setting.

In this paper the main focus is on the use of plantar force measurement in the recognition of main postures from activities of daily life. We propose a preliminary study to find optimal sensor locations under the foot that estimate total force. The output of the best force estimator is then used to assess the accuracy of our activity recognition algorithm (based on linear classifiers [9]). The objective of the experiments is twofold: ( $i$ ) to verify the hypothesis that the total force can be accurately estimated using only two sensors and (ii) to verify that the activity/posture recognition in this context could be accurately assessed from the estimated forces.

\section{MATERIALS AND METHODS}

Five subjects, aged $=25.6 \pm 2.3$ years, weight $=66.4 \pm 12.9 \mathrm{~kg}$, were asked to perform a series of activities including sitting (on three different types of chairs), standing (including bending to pick up objects from floor), and walking (level, upstairs and downstairs). Each subject wore a commercial pair of force sensing insoles chosen as reference (Pedar, Novel, Germany). The insole's dense mesh of individual sensors was used to recalculate the measurable force at any placement and for each size of our proposed combinations. Force data were collected via the data logger worn in a belt by each subject, sampled at $100 \mathrm{~Hz}$. The trials were video recorded for activity labeling. Each trial lasted 5 to 6 minutes. 


\section{A. Optimal sensor configuration}

\section{1) Sensor configuration}

First, we divided the foot into forefoot and rear foot sections. The division of the foot into two or more segments has been previously addressed in the literature [10], [11]. The goal here was to find the best location in each section for total force estimate. The measurable force for each sensor placement was estimated (calculated) based on the individual cells in the reference insole system, see Figure 1.

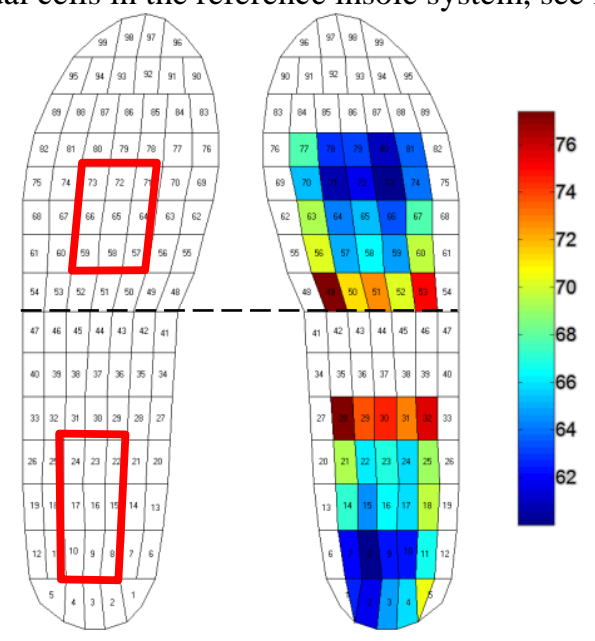

Figure 1-Pedar cells on each insole. Left: The red squares represent the best locations found with our force estimator. Right: color map of errors (N) when the best locations are moved by one cell around the center of the best location.

We considered rectangular shapes and three sensor sizes which were tested based on the selection of number of cells $(2 \times 3,3 \times 3$ and $3 \times 4)$. For each size and insole the number of possible different locations was estimated in each foot section $\left(N_{\text {fore }}, N_{\text {rear }}\right)$. The total sensor force $\left(\widehat{F}_{\text {rear }}, \hat{F}_{\text {fore }}\right)$ for each size $(i=1,2,3)$ and location $\left(n_{r}=\right.$ $\left.1, \cdots, N_{\text {rear }}, n_{f}=1, \cdots, N_{\text {fore }}\right)$ was estimated by the sum of the corresponding cells according to equation 1 :

$\hat{F}_{\text {rear }}=\sum_{i, n_{r}} F_{i, n_{r}}$ and $\hat{F}_{\text {fore }}=\sum_{i, n_{f}} F_{i, n_{f}}$

\section{2) Estimation of total force and validation}

The data from all the five subjects were split into training and testing: a subset from each subject containing data from sitting, standing and walking activities was included in the training set, with the rest of the data (still containing all the activities) used for testing. The training data were used to build the estimators of total force and the test data to evaluate the best estimators. We tested all the possible combinations of fore and rear foot locations for each size. A linear regression model [12] as shown in equation 2 was used to estimate the total force based on calculated forces from each combination of fore and rear sensor locations for each of the three sensor sizes:

$$
\hat{F}_{\text {total }}=w_{\text {offset }}+w_{\text {fore }} \times \hat{F}_{\text {fore }}+w_{\text {rear }} \times \hat{F}_{\text {rear }}
$$

Where $w_{\text {offset }}, w_{\text {fore }}$ and $w_{\text {rear }}$ are the weights of the linear regression model obtained via the least square error solution on the training data, and $\widehat{F}_{\text {total }}$ is the estimated total force. The best configuration was that with the lowest root mean square error (RMSe). The force estimate based on this configuration was fed into an activity recognition algorithm detailed in the next section.

\section{B. Activity recognition}

\section{1) Classification algorithm}

This section describes the activity recognition algorithm that was employed to classify the different activities using the estimated forces (left and right feet) from the selected sensor configuration. The classification algorithm was designed to enable the distinction between three different activities: sitting, standing and walking; climbing up and down the stairs was categorized as walking whereas picking an object from the floor as standing. We studied the effect of the subject and environment based factors such as subjects' weights and chair types on the classifier. The four parameters of the activity classifier were: subject's weight (SW - continuous), the chair type (CT, discrete: from 1 to 3 upon chair types), the estimated force from the right insole (RF - continuous), and the estimated force from the left insole (LF - continuous). Then the effect of each parameter and its interaction with other parameters were studied using one-way ANOVA [13] on the training data sets. Activities were labeled as: $0=$ sitting, $1=$ standing and $2=$ walking. A linear model (equation 3) was learned on the training sets.

Actıvity $=\gamma+\sum_{i} \alpha_{i} \times X_{i}+\sum_{i, j \text { relevant }} \beta_{i j} \times X_{i} * X_{j}$

Where $\mathrm{Xi}$ and $\mathrm{Xj}$ are the previously described parameters. Actıvity represents the predicted activity as a combination of $\gamma$ (the intercept), $\alpha$ (the first order coefficient) and $\beta$ (the interactions coefficient); rounded to the nearest integer (equivalent to two-level thresholding), i.e. the activity label.

\section{2) Validation}

The reference labels for activities were obtained via automatic labeling based on the reference total force from all 99 sensors in Pedar insoles, cross-checked with video recordings. To do so, two thresholds were used to separate the different activities based on video tagging. Parameters with a p-value smaller than $1 \%$ were considered as relevant. The predicted activities were compared with the reference activity labels to find the best recognition rate. The most relevant parameters were included in the final model and the recognition rate was also computed on the entire data sets ( $80 \%$ of which is test which that was not included for the ANOVA) for continuity purposes. It must be noted that, by using a 2-second window (corresponding to 200 samples), we split the data to the events to be labeled for activity recognition. The median values of the estimated total force in each event were chosen for classification. 
The correct classification rate (CCR) was defined as the number of events classified correctly to activities over the number of events (across all subjects).

$$
C C R=\frac{\text { number of correctly recognized activities }}{\text { number of samples }}
$$

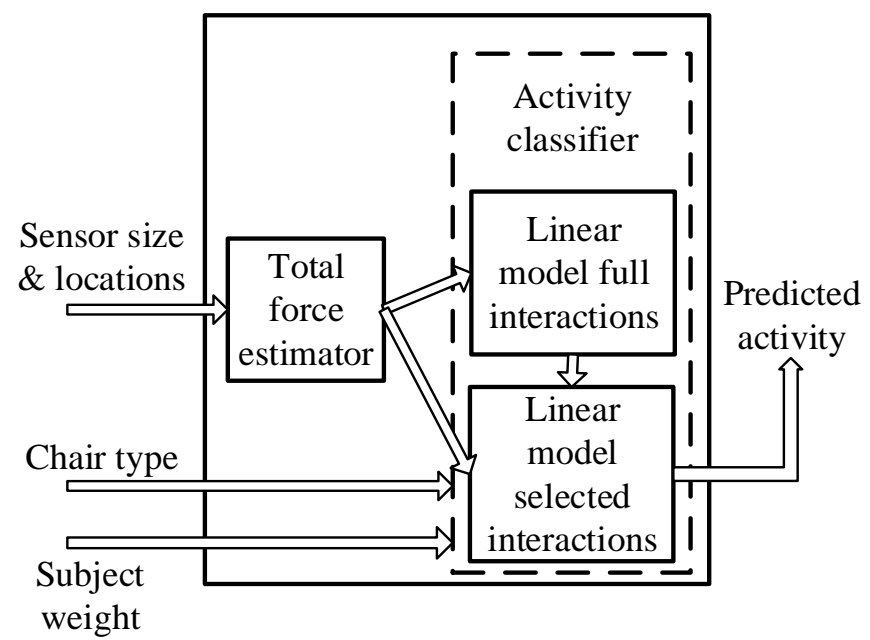

Figure 2-Activity classification diagram showing the inputs and output of the classifier

Figure 2 summarizes the method for the activity classification and the effects of the different inputs.

\section{RESULTS}

\section{A. Selection of shape, size and location}

Table 1 details the number of possible locations for fore and rear sensor placements for each size, and the minimum RMS error achieved in all the simulations. Based on the minimum RMS error for test data, the best force estimate was obtained from the $3 \times 3$ rectangle (type 2) with the location combination illustrated by the red rectangles in Figure 1. The linear regression weights (offset and gains) in Eq. 3 were computed from the training data (concatenation of all subjects training data) and presented in equation 4 .

$$
\hat{F}=3.01+2.22 \times \widehat{F}_{\text {rear,size } 2}+3.06 \times \widehat{F}_{\text {fore,size } 2}
$$

Also shown in Figure 1 are the errors that would occur of the best placement is shifted by 1 cell around the center of the best location.

Table 1-Total number of possible combinations for simulation

\begin{tabular}{|c|c|c|c|}
\hline Shape & Rectangle & Rectangle & Rectangle \\
\hline Size (cells) & $2 \times 3$ & $3 \times 3$ & $3 \times 4$ \\
\hline \# fore locations & 34 & 30 & 24 \\
\hline \# rear location & 30 & 25 & 20 \\
\hline \# combinations & 1020 & 750 & 480 \\
\hline Min RMSe (N) & 47.1 & 39.9 & 41.3 \\
\hline
\end{tabular}

This was a result of fixing one position in one section (fore or rear) and averaging the error from all the combinations of this position with the available positions in the other section.

\section{B. Physical activity recognition}

\section{1) Finding the most relevant factors}

A one-way ANOVA was used to find the most relevant factors affecting PA classification. Table 2 displays the results of the ANOVA. The degrees of freedom of continuous parameters were set to one whereas the discrete parameter CT (3 types of chair) was set to 2 . Relevant parameters $(\mathrm{p}<0.01)$ are the left force $(\mathrm{LF})$, the right force $(\mathrm{RF})$ and the interaction between the two $(\mathrm{LF} \times \mathrm{RF})$ forming equation 5. The type of chair and the subject's weight do not have a significant effect on the activity classifier.

Table 2 - ANOVA results. Parameters are : RF : estimated force from the right insole / LF: estimated force from the left insole / SW subject's weight / CT :chair type. $\mathbf{M}_{\%}$ denotes the percentage of the effect mean squares over the total squares. Mean Sq is SumSq divided by the degrees of freedom and

\begin{tabular}{lrrrrr}
\multicolumn{7}{c}{ F is the F test. } \\
\hline \hline SumSq & DF & MeanSq & F & p \\
RF & 41.00 & 1 & 41.00 & 310.98 & $<0.01$ \\
CT & 43.55 & 1 & 43.55 & 330.31 & $<0.01$ \\
SW & 0.16 & 2 & 0.08 & 0.59 & 0.56 \\
LF x RF & 0.24 & 1 & 0.24 & 1.81 & 0.18 \\
LF x CT & 13.79 & 1 & 13.79 & 104.61 & $<0.01$ \\
LF x SW & 0.08 & 2 & 0.04 & 0.30 & 0.74 \\
RF x CT & 0.11 & 1 & 0.11 & 0.80 & 0.37 \\
RF x SW & 0.23 & 2 & 0.11 & 0.85 & 0.43 \\
CT x SW & 0.02 & 1 & 0.02 & 0.13 & 0.72 \\
Error & 0.28 & 2 & 0.14 & 1.06 & 0.35 \\
Total & $\mathbf{3 0 . 0 6}$ & $\mathbf{2 2 8}$ & $\mathbf{0 . 1 3}$ & & \\
\hline \hline
\end{tabular}

Upon finding the most relevant parameters, the linear model used as the final classifier is presented in equation 5 .

Actlvity $=\gamma+\alpha_{1} \times L F+\alpha_{2} \times R F+\beta_{12} L F * R F$

Where $\gamma=1.16$. The contribution of the interaction between $\mathrm{LF}$ and RF was substantial $\left(\beta_{12}=-0.26\right)$ to counterbalance the $\operatorname{LF}\left(\alpha_{1}=0.27\right)$ and $\operatorname{RF}\left(\alpha_{2}=0.30\right)$.

2) CCR, Sensitivity and Specificity of the activity recognition algorithm

Table 3- Confusion matrix and sensitivity and specificity values

\begin{tabular}{|l|r|r|r||r|}
\hline $\begin{array}{l}\text { Actual } \rightarrow \\
\text { Predicted }\end{array}$ & \multicolumn{1}{|l|}{ Sit } & \multicolumn{1}{|l|}{ Stand } & \multicolumn{1}{l|}{ Specificity } \\
\hline Sit & 93.83 & 0.01 & 0 & $\mathbf{0 . 9 8 8 4}$ \\
\hline Stand & 5.93 & 99.54 & 6.64 & $\mathbf{0 . 9 9 7 3}$ \\
\hline Walking & 0.24 & 0.45 & 93.36 & $\mathbf{0 . 9 3 3 6}$ \\
\hline \hline Sensitivity & $\mathbf{0 . 9 3 8 2}$ & $\mathbf{0 . 9 9 5 4}$ & $\mathbf{0 . 9 3 3 6}$ & $\mathbf{9 5 . 6 4}$ \\
\hline
\end{tabular}

In Table 3, the confusion matrix [9], representing how different activities are recognized, shows that the activities are accurately recognized with an average CCR of $95.64 \%$ and all the diagonal values of the confusion matrix are 
greater than $90 \%$. However, sitting and walking was sometimes classified as standing. Furthermore, the sensitivity and specificity were calculated for all activities classifications and presented in Table 3 as percentage. Figure 3 depicts a sample output of our classifier algorithm.

\section{DISCUSSION AND CONCLUSION}

In this study, we proposed a minimal force sensor configuration to appropriately estimate the total force and to recognize basic activities of daily life. The best locations for sensor placement in this study were found with a relatively small RMS error $(40 \mathrm{~N})$. The error of total force estimation is also low in many regions close to the best locations as seen in Figure 1. This would reduce the effect of slight misplacement or sensor slippage in the shoes on the estimation of total force. The success of our simple activity classification approach was confirmed with the high CCR and the negligible difference in CCR (only increased by $0.5 \%$ ) when reference force data was used (as opposed to estimated data from the first analysis). We hypothesized that weight and chair type will have an effect on the activity recognition, without having prior knowledge about these effects. We concluded that the effect of these factors was not significant. This provides an advantage since the algorithm needs no tuning with respect to individual weight or chair type. However, more environmental and subject related factors need to be studied such as gait patterns and floor types in order to have a clearer conclusion on the effect of individual and environmental factors. Additionally, the small number of subjects needs to be increased for more conclusive results. We are also aware that other studies have obtained activity recognition with similar accuracies while using more sensors. However, the novelty of our approach was in finding the best locations for force estimation first. Furthermore, using only force signals, we obtained high accuracies via a simple classifier. The classification was based on linear regression because this way we were able to assess the effects of different factors using ANOVA. We expect to increase this accuracy by adding inertial sensors to our shoe-based system, and employing more sophisticated classifiers.

In our case, the algorithm was straightforward for the detection of simple activities. We grouped some activities under the same label even though they were different; however, our results are very encouraging for detection of additional activities such as climbing up and down the stairs and picking an object, and this will be done in a later stage by adding inertial sensing. On the other hand, the activities in our protocol were performed in controlled conditions; however the subjects chose how to perform the activities (self-selected speed, time of sit and stand). In subsequent work, the focus will be on real world conditions without any constraint in order to have a more realistic approach for activity classification. This work is part of an on-going larger activity recognition study in which we will fuse additional inputs from other sensors such as inertial measurement units to achieve highly accurate and robust recognition of at home, free daily life activities.

\section{ACKNOWLEDGMENTS}

We would like to acknowledge the EC fund GA (288940) as this work is part of the FP7 FARSEEING project.

Activity recognition output

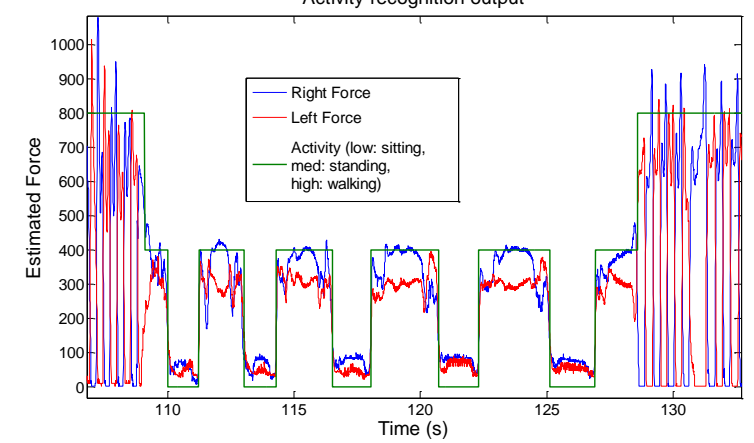

Figure 3- Output of the activity recognition algorithm

\section{REFERENCES}

[1] C.-C. Yang and Y.-L. Hsu, "A review of accelerometry-based wearable motion detectors for physical activity monitoring.," Sensors (Basel), vol. 10, no. 8, pp. 7772-88, Jan. 2010.

[2] E. S. Sazonov, G. Fulk, J. Hill, Y. Schutz, and R. Browning, "Monitoring of posture allocations and activities by a shoe-based wearable sensor.," IEEE Trans Biomed Eng, vol. 58, no. 4, pp. 983-90, Apr. 2011.

[3] X. Zhang, Y. Zhao, Z. Duan, and Y. Liu, "Design and test of a soft plantar force measurement system for gait detection.," Sensors (Basel), vol. 12, no. 12, pp. 16628-40, Jan. 2012.

[4] J. Cobb and D. J. Claremont, "Transducers for foot pressure measurement: survey of recent developments," Med Biol Eng Comput, no. 33, pp. 525-532, 1995.

[5] S. M. Bamberg, P. Lastayo, L. Dibble, J. Musselman, and S. K. D. Raghavendra, "Development of a quantitative in-shoe measurement system for assessing balance: sixteen-sensor insoles.," in Conf Proc IEEE Eng Med Biol Soc, 2006, vol. 1, pp. 6041-4.

[6] M. Benocci, L. Rocchi, E. Farella, L. Chiari, and L. Benini, “A wireless system for gait and posture analysis based on pressure insoles and Inertial Measurement Units," in Int Conf Pervasive Comput Technol Healthc, 2009.

[7] R. Lvping, L. Deyu, L. Chengrui, Y. Yang, Q. Yajun, Y. Songyan, P. Fang, and N. Haijun, "Design of in-shoe plantar pressure monitoring system for daily activity exercise stress assessment," Proc 4th Int Conf Biomed Eng Inform, pp. 13671370, Oct. 2011.

[8] S. Pfaffen, P. Sommer, C. Stocker, R. Wattenhofer, and S Welten, "Planipes : Mobile Foot Pressure Analysis," in mHealthSys, 2011.

[9] R. O. Duda, Pattern Classification, 2nd Ed. John Wiley \& Sons Asia PTE, 2007.

[10] A. Caselli, H. Pham, J. M. Giurini, D. G. Armstrong, and A. Veves, "The forefoot-to-rearfoot plantar pressure ratio is increased in severe diabetic neuropathy and can predict foot ulceration.," Diabetes care, vol. 25, no. 6, pp. 1066-71, Jun. 2002.

[11] H. Rouhani, X. Crevoisier, J. Favre, and K. Aminian, "Outcome evaluation of ankle osteoarthritis treatments: plantar pressure analysis during relatively long-distance walking.," Clin Biomech (Bristol, Avon), vol. 26, no. 4, pp. 397-404, May 2011.

[12] O. Nelles, Nonlinear System Identification: From Classical Approaches to Neural Networks and Fuzzy Models. Springer, 2001.

[13] R. V Hogg and J. Ledolter, Engineering Statistics. Macmillan Publ.Comp., 1989. 